\title{
Six-Quark Amplitudes from Fermionic MHV Vertices
}

\author{
Xun $\mathrm{Su}^{*}$ \\ Institute of Theoretical Physics, Chinese Academy of Sciences \\ P. O. Box 2735, Beijing 100080, P. R. China \\ e-mail: ghkcn@itp.ac.cn \\ Jun-Bao Wu \\ School of Physics, Peking University \\ Beijing 100871, P. R. China \\ e-mail: jbwu@pku.edu.cn
}

July 16, 2018

\begin{abstract}
The fermionic extension of the CSW approach to perturbative gauge theory coupled with fermions is used to compute the six-quark QCD amplitudes. We find complete agreement with the results obtained by using the usual Feynman rules.
\end{abstract}

*Supported in part by fund from the National Natural Science Foundation of China with grant Number 90103004. 


\section{Introduction}

Recently Witten [1] found a deep connection between the perturbative gauge theory and string theory in twistor space [2]. Based on this work, Cachazo, Svrcek and Witten (CSW for short) reformulated the perturbative calculation of the scattering amplitudes in Yang-Mills theory using the off shell MHV vertices [3]. The MHV vertices are the usual tree level MHV scattering amplitudes in gauge theory [4, 5], continued off shell in a particular fashion as given in [3]. (For references on perturbative calculations, see for example [6- [10]. The 2 dimensional origin of the MHV amplitudes in gauge theory was first given in [11.) Some sample calculations were done in [3, sometimes with the help of symbolic manipulation. The correctness of the rules was partially verified by reproducing the known results for small number of gluons [6].

In a previous work [12] (for recent works, see [13]-36]), the extension of the CSW approach to theories with fermions (see also [23]) is discussed and used to calculate the googly fermionic amplitudes. The amplitudes with 3 quark-anti-quark pairs which are neither MHV nor googly were also analyzed in that paper. In this paper, we will calculate these amplitudes explicitly by using the CSW rules and show that the results are in agreement with the results obtained by using the usual Feynman rules [37. Although some generic non-MHV fermionic amplitudes were also calculated in [23, 33], we found that it is also worthy to do this calculation. As we will see in the following, the calculation by using the usual Feynman rules is even simpler. So the purpose of our paper is actually to check that the MHV rules are really correct in this non-trivial case.

The MHV (and googly) amplitudes with gluinos or one quark-anti-quark pair can be obtained from the gluonic amplitudes via supersymmetric Ward identities (SWI's) 38, 39, 6]. But there are more fermionic amplitudes which cannot be obtained in this way. In [40], it has been discussed that neither the non-MHV (googly) amplitudes with gluinos nor the MHV (googly) amplitudes with two quark-anti-quark pairs can be obtained from the gluonic amplitudes by using the SWI's (See also [33]). In some sense the amplitudes which cannot be obtained via SWI's have more information. So it is worthy to calculate these amplitudes by using the CSW rules. Although the CSW rule can be partially understood from the twistor string theory [24, a full understanding of the CSW approach from the conventional field theory is not reached [29].

This paper is organized as follows. In section 2, we first review CSW 
rules for gauge theory without fermions. Then we review extended CSW rules for gauge theory with quarks and the analysis on the CSW diagrams for six-quark amplitudes. In section 3, we calculate the amplitudes with 3 quark-anti-quark pairs by using the fermionic extension of CSW rules. We show that the result coincides with which from Feynman rules.

\section{CSW rules with fermions}

First let us review the rules for calculating tree level gauge theory gluonic amplitudes proposed in [3]. Here we follow the presentation given in [13, 14, 12 closely, and consider only partial amplitudes 6]. We will use the convention that all momenta are outgoing. By MHV (with gluons only), we always mean an amplitude with precisely two gluons of negative helicity. If the two gluons of negative helicity are labelled as $r, s$, the MHV vertices are given as follows:

$$
V_{n}=\frac{\left\langle\lambda_{r}, \lambda_{s}\right\rangle^{4}}{\prod_{i=1}^{n}\left\langle\lambda_{i}, \lambda_{i+1}\right\rangle} .
$$

For an on shell (massless) gluon, the momentum in bispinor basis is given as:

$$
p_{a \dot{a}}=\sigma_{a \dot{a}}^{\mu} p_{\mu}=\lambda_{a} \tilde{\lambda}_{\dot{a}}
$$

For an off shell momentum, we can no longer define $\lambda_{a}$ as above. The offshell continuation given in [3] is to choose an arbitrary spinor $\tilde{\eta}^{\dot{a}}$ and then to define $\lambda_{a}$ as follows:

$$
\lambda_{a}=p_{a \dot{a}} \tilde{\eta}^{\dot{a}} .
$$

For an on shell momentum $p$, we will use the notation $\lambda_{p a}$ which is proportional to $\lambda_{a}$ :

$$
\lambda_{p a} \equiv p_{a \dot{a}} \tilde{\eta}^{\dot{a}}=\lambda_{a} \tilde{\lambda}_{\dot{a}} \tilde{\eta}^{\dot{a}} \equiv \lambda_{a} \phi_{p}
$$

As demonstrated in [3], it is consistent to use the same $\tilde{\eta}$ for all the off shell momenta. The final result is independent of $\tilde{\eta}$.

By using only MHV vertices, one can build a tree diagram by connecting MHV vertices with propagators. For the propagator of momentum $p$, we assign a factor $1 / p^{2}$. The helicity at two ends of a propagator must be opposite. Any possible diagram with the color factor $\operatorname{Tr}\left(T^{a_{1}} \cdots T^{a_{n}}\right)$ contributes to the partial amplitude $A_{n}\left(g_{1}^{h_{1}}, \cdots, g_{n}^{h_{n}}\right)$.

Now we review the extension of CSW rules to the gauge theory coupled with quarks and anti-quarks [23, 12. For this theory we can decompose an 
amplitude into partial amplitudes with definite color factors [6]. For simplicity we will assume that all quarks have different flavors. When there are identical quarks, the amplitudes can be easily obtained from the amplitudes with distinct quarks. Also we will assume the gauge group to be $U(N)$ instead of $S U(N)$. For a connected diagram with $m$ pair of quark-antiquark, the color factor is

$$
\left(T^{a_{1}} \cdots T^{a_{n_{1}}}\right)_{i_{i_{1}} \overline{i_{2}}}\left(T^{a_{n_{1}+1}} \cdots T^{a_{n_{2}}}\right)_{i_{2} \overline{i_{3}}} \cdots\left(T^{a_{n_{m-1}+1}} \cdots T^{a_{n}}\right)_{i_{m} \overline{i_{1}}},
$$

for a particular ordering of the quark-antiquarks and gluons [41]. For amplitudes with connected Feynman diagrams, the quark-antiquark color indices $(i, \bar{i})$ must form a ring of length exactly $m$. This can be proved by induction with the number of pairs $m$.

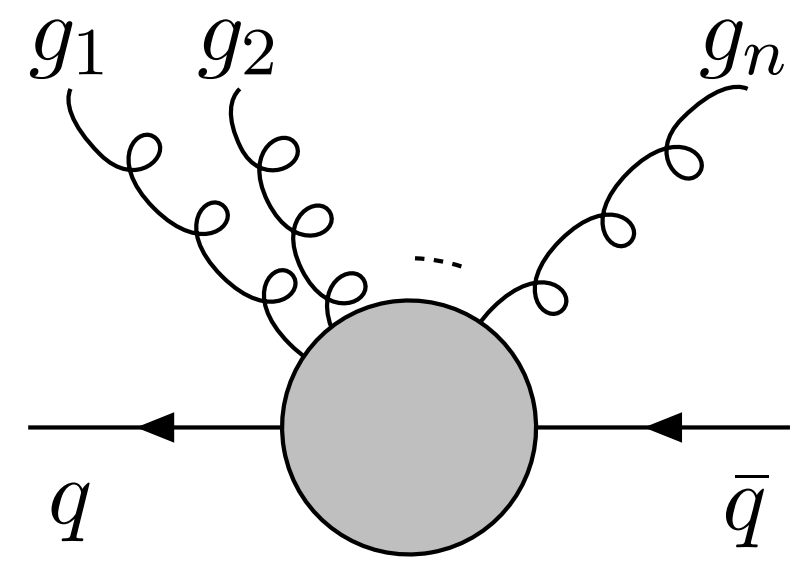

Figure 1: The graphic representation for the single pair of quark-antiquark partial amplitude. Gluons are emitted from one side of the fermion line only.

For a single quark-antiquark pair the color factor is $\left(T^{a_{1}} \cdots T^{a_{n}}\right)_{i \bar{i}}$. The partial amplitude is denoted as $A_{n+2}\left(\Lambda_{q}^{h}, g_{1}, \cdots, g_{n}, \Lambda_{\bar{q}}^{-h}\right)^{1}$. It is represented as in Fig. 1. We note that gluon lines are emitted only from one side of the (connected) quark-anti-quark line. We will stick to this rule also for multi-pair of quark-antiquark diagrams.

There are $2 \mathrm{MHV}$ vertices with quark-anti-quarks, one for a single pair of quark-antiquark and one for two quark-anti-quark pairs which are shown in

\footnotetext{
${ }^{1} h$ denotes the helicity of the quark $q$. The helicity of the antiquark $\bar{q}$ is $-h$ by helicity conservation along the quark line.
} 

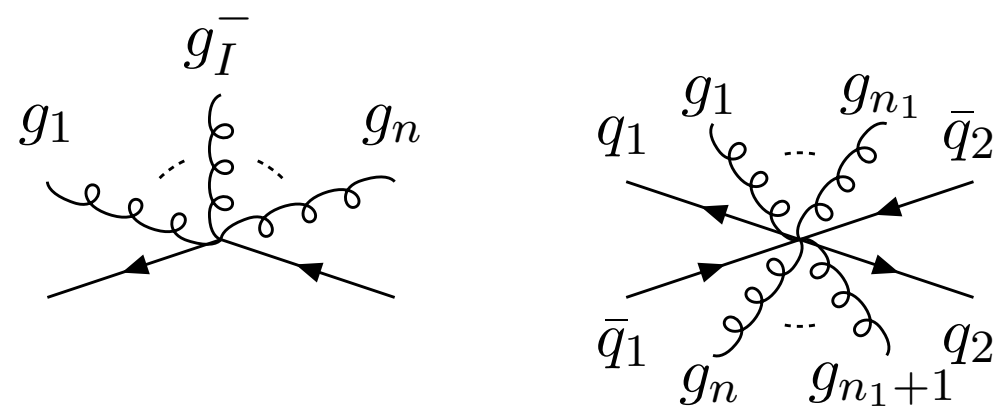

Figure 2: The $2 \mathrm{MHV}$ vertices with quark-antiquarks.

Fig. 2. There is no MHV vertex for 3 or more pair of quark-antiquark. All these (non-MHV) amplitudes should be computed from the above MHV vertices by drawing all possible (connected) diagrams with only MHV vertices.

The explicit formulas for these MHV vertices (or amplitudes) are given as follows:

$$
\begin{aligned}
V\left(\Lambda_{q}^{+}, g_{1}^{+}, \cdots, g_{I}^{-}, \cdots, g_{n}^{+}, \Lambda_{\bar{q}}^{-}\right) & =-\frac{\langle q, I\rangle\langle\bar{q}, I\rangle^{3}}{\langle q, 1\rangle\langle 1,2\rangle \cdots\langle n, \bar{q}\rangle\langle\bar{q}, q\rangle}, \\
V\left(\Lambda_{q}^{-}, g_{1}^{+}, \cdots, g_{I}^{-}, \cdots, g_{n}^{+}, \Lambda_{\bar{q}}^{+}\right) & =\frac{\langle q, I\rangle^{3}\langle\bar{q}, I\rangle}{\langle q, 1\rangle\langle 1,2\rangle \cdots\langle n, \bar{q}\rangle\langle\bar{q}, q\rangle},
\end{aligned}
$$

for the single pair of quark-antiquark, and for 2 quark-antiquark pairs:

$$
\begin{aligned}
& V\left(\Lambda_{q_{1}}^{h_{1}}, g_{1}, \cdots, g_{n_{1}}, \Lambda_{\bar{q}_{2}}^{-h_{2}}, \Lambda_{q_{2}}^{h_{2}}, g_{n_{1}+1}, \cdots, g_{n}, \Lambda_{\bar{q}_{1}}^{-h_{1}}\right) \\
& \quad=A_{0}\left(h_{1}, h_{2}\right) \frac{\left\langle q_{1}, \bar{q}_{2}\right\rangle}{\left\langle q_{1}, 1\right\rangle\langle 1,2\rangle \cdots\left\langle n_{1}, \overline{q_{2}}\right\rangle} \times \frac{\left\langle q_{2}, \bar{q}_{1}\right\rangle}{\left\langle q_{2}, n_{1}+1\right\rangle \cdots\left\langle n, \bar{q}_{1}\right\rangle},
\end{aligned}
$$

where $A_{0}\left(h_{1}, h_{2}\right)$ is given as follows:

$$
\begin{array}{cc}
A_{0}(+,+)=\frac{\left\langle\bar{q}_{1}, \bar{q}_{2}\right\rangle^{2}}{\left\langle q_{1}, \bar{q}_{1}\right\rangle\left\langle q_{2}, \bar{q}_{2}\right\rangle}, & A_{0}(+,-)=-\frac{\left\langle\bar{q}_{1}, q_{2}\right\rangle^{2}}{\left\langle q_{1}, \bar{q}_{1}\right\rangle\left\langle q_{2}, \bar{q}_{2}\right\rangle}, \\
A_{0}(-,+)=-\frac{\left\langle q_{1}, \bar{q}_{2}\right\rangle^{2}}{\left\langle q_{1}, \bar{q}_{1}\right\rangle\left\langle q_{2}, \bar{q}_{2}\right\rangle}, & A_{0}(-,-)=\frac{\left\langle q_{1}, q_{2}\right\rangle^{2}}{\left\langle q_{1}, \bar{q}_{1}\right\rangle\left\langle q_{2}, \bar{q}_{2}\right\rangle} .
\end{array}
$$

All these MHV amplitudes are given in terms of the "holomorphic" spinors of the external (on-shell) momenta. So we can use the same off shell continuation given in [3] which we recalled above. By including these fermionic 
MHV vertices, we can extend the CSW rule of perturbative gauge theory to gauge theories with quarks and anti-quarks. The propagator for both gluon and fermion (quark or anti-quark) internal lines is just $1 / p^{2}$, as explained in [23. Helicity is conserved along a fermion line. Because we assume that all quarks have different flavor, the flowing of arrows must follow the directions given exactly in Fig. 2. We use the rules given in [23] to sew vertices by propagators.

Assume that in an MHV diagram, there are $n_{i}$ purely gluonic MHV vertices with $i$ lines, $n_{i}^{2 f}$ single pair of quark-antiquark MHV vertices with $(i+2)$ lines (counting also the 2 fermion lines, so actually only $i$ gluon lines), and $n_{i}^{4 f} 2$ pairs of quark-antiquark MHV vertices with $(i+4)$ lines (counting also the 4 fermion lines, so actually only $i$ gluon lines). For a connected diagram with $m$ quark-antiquark pairs, the number of positive helicity gluon $n_{+}$and the number of negative helicity gluon $n_{-}$are given as follows [12:

$$
\begin{aligned}
& n_{-}=\sum_{i \geq 3} n_{i}+\sum_{i \geq 1} n_{i}^{2 f}+\sum_{i \geq 0} n_{i}^{4 f}-(m-1), \\
& n_{+}=\sum_{i \geq 3}(i-3) n_{i}+\sum_{i \geq 1}(i-1) n_{i}^{2 f}+\sum_{i \geq 0}(i+1) n_{i}^{4 f}-(m-1) .
\end{aligned}
$$

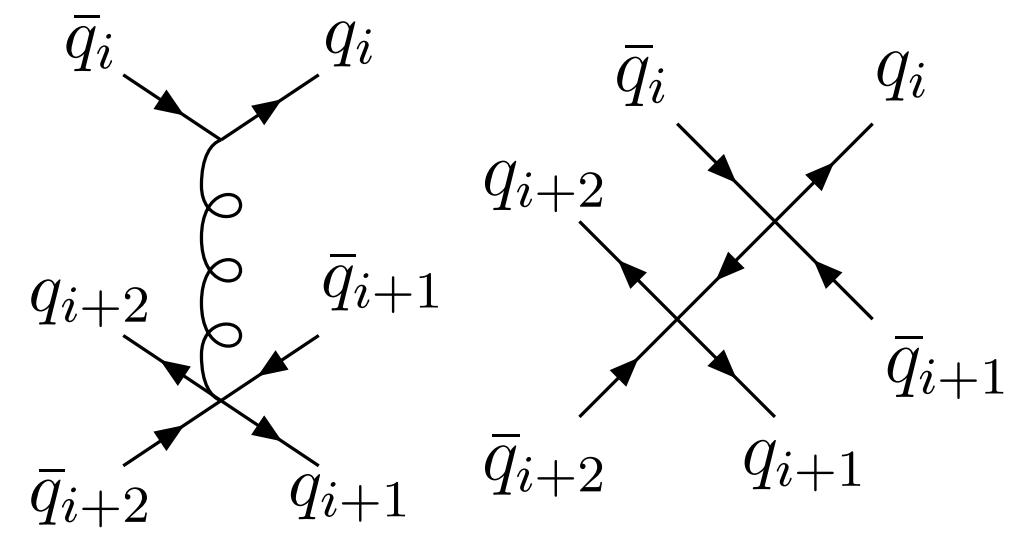

Figure 3: The 2 different kinds of CSW diagrams contributing to the purely fermionic amplitude with 3 quark-antiquark pairs. In these diagrams $i$ can take 1,2,3 and the indices are understood as mod 3 . So totally there are 6 diagrams.

These relations are particularly useful for analyzing diagrams with fewer 
number of external gluons with positive helicity. For the purely fermionic amplitudes with 3 quark-antiquark pairs, there are only 2 different kinds of diagrams as shown in Fig. 3 12. By using the extended CSW rules, the partial amplitude can be written down very simply. We show in the next section that this gives the right result for the amplitude.

\section{The purely fermionic amplitudes with three quark-antiquark pairs}

As mentioned in the previous section, we will compute the purely fermionic amplitudes with three distinct (massless) quark-anti-quark pairs. The color factor is $\delta_{i_{1} \bar{i}_{2}} \delta_{i_{2} \bar{i}_{3}} \delta_{i_{3} \bar{i}_{1}}$, for a particular ordering of the quark-antiquarks. The partial amplitude is denoted as $A_{6}\left(\Lambda_{q_{1}}^{h_{1}}, \Lambda_{\bar{q}_{2}}^{-h_{2}}, \Lambda_{q_{2}}^{h_{2}}, \Lambda_{\bar{q}_{3}}^{-h_{3}}, \Lambda_{q_{3}}^{h_{3}}, \Lambda_{\bar{q}_{1}}^{-h_{1}}\right)$.

In this section, we will compute these partial amplitudes first by using the CSW rules, then by using the Feynman rules. we will show that these two results coincite up to a phase factor because of the phase convention we used for the vertices and the propagators in the CSW approach.

As mentioned above, in the CSW approach to compute this partial amplitudes, there are only 2 different kinds of CSW diagrams as shown in Fig. 3 (We note that these CSW diagrams corresponding to the amplitudes with different helicity configurations are the same. This is different from many gluonic amplitudes, where the CSW diagrams corresponding to the amplitudes with different helicity configurations are different.)

There are 8 kinds of helicity configurations. We can find some relations among these amplitudes with different helicity configurations.

This first relation is:

$$
A_{6}\left(\Lambda_{q_{1}}^{h_{1}}, \Lambda_{\bar{q}_{2}}^{-h_{2}}, \Lambda_{q_{2}}^{h_{2}}, \Lambda_{\bar{q}_{3}}^{-h_{3}}, \Lambda_{q_{3}}^{h_{3}}, \Lambda_{\bar{q}_{1}}^{-h_{1}}\right)=A_{6}\left(\Lambda_{q_{2}}^{h_{2}}, \Lambda_{\bar{q}_{3}}^{-h_{3}}, \Lambda_{q_{3}}^{h_{3}}, \Lambda_{\bar{q}_{1}}^{-h_{1}}, \Lambda_{q_{1}}^{h_{1}}, \Lambda_{\bar{q}_{2}}^{-h_{2}}\right) .
$$

This means that the amplitudes are invariant under the cyclic permutation of the quark-anti-quark pairs.

The second one is the relation between the two amplitudes related by charge conjugation:

$$
A_{6}\left(\Lambda_{q_{1}}^{h_{1}}, \Lambda_{\bar{q}_{2}}^{-h_{2}}, \Lambda_{q_{2}}^{h_{2}}, \Lambda_{\bar{q}_{3}}^{-h_{3}}, \Lambda_{q_{3}}^{h_{3}}, \Lambda_{\bar{q}_{1}}^{-h_{1}}\right)=-A_{6}\left(\Lambda_{q_{3}^{\prime}}^{-h_{3}}, \Lambda_{\bar{q}_{2}^{\prime}}^{h_{2}}, \Lambda_{q_{2}^{\prime}}^{-h_{2}}, \Lambda_{\bar{q}_{1}^{\prime}}^{h_{1}}, \Lambda_{q_{1}^{\prime}}^{-h_{1}}, \Lambda_{\bar{q}_{3}^{\prime}}^{h_{3}}\right)
$$

where $\lambda_{q_{i}^{\prime}}=\lambda_{\bar{q}_{i}}, \lambda_{\bar{q}_{i}^{\prime}}=\lambda_{q_{i}}, \tilde{\lambda}_{q_{i}^{\prime}}=\tilde{\lambda}_{\bar{q}_{i}}, \tilde{\lambda}_{\bar{q}_{i}^{\prime}}=\tilde{\lambda}_{q_{i}}$. 
These relations can be easily verified case by case, either using the CSW rules or using the Feynman rules.

So we only need to consider the case when $h_{1}=h_{2}=h_{3}=-1 / 2$ and the case when $h_{1}=h_{2}=-1 / 2, h_{3}=1 / 2$. Other cases can be obtained from these cases by permutation of the quark-anti-quark pairs and/or charge conjugation transformation.

When $h_{1}=h_{2}=h_{3}=-1 / 2$, the amplitude is:

$$
A_{6}^{C S W}\left(\Lambda_{q_{1}}^{-}, \Lambda_{\bar{q}_{2}}^{+}, \Lambda_{q_{2}}^{-}, \Lambda_{\bar{q}_{3}}^{+}, \Lambda_{q_{3}}^{-}, \Lambda_{\bar{q}_{1}}^{+}\right)=\sum_{i=1}^{3} A^{i}+\sum_{i=1}^{3} \tilde{A}^{i},
$$

where

$$
\begin{aligned}
A^{i}= & -\frac{\left\langle q_{i},\left(\bar{q}_{i} q_{i}\right)\right\rangle^{3}\left\langle\bar{q}_{i},\left(\bar{q}_{i} q_{i}\right)\right\rangle}{\left\langle\bar{q}_{i}, q_{i}\right\rangle\left\langle q_{i},\left(\bar{q}_{i} q_{i}\right)\right\rangle\left\langle\left(\bar{q}_{i} q_{i}\right), \bar{q}_{i}\right\rangle} \frac{1}{\left(p_{\bar{q}_{i}}+p_{q_{i}}\right)^{2}} \\
& \times \frac{\left\langle q_{i+1}, q_{i+2}\right\rangle^{2}}{\left\langle\bar{q}_{i+1}, q_{i+1}\right\rangle\left\langle\bar{q}_{i+2}, q_{i+2}\right\rangle} \frac{\left\langle q_{i+2}, \bar{q}_{i+1}\right\rangle}{\left\langle q_{i+2},\left(\bar{q}_{i} q_{i}\right)\right\rangle\left\langle\left(\bar{q}_{i} q_{i}\right), \bar{q}_{i+1}\right\rangle} \\
= & \frac{\left\langle q_{i},\left(\bar{q}_{i} q_{i}\right)\right\rangle^{2}}{\left\langle\bar{q}_{i}, q_{i}\right\rangle} \frac{1}{\left(p_{\bar{q}_{i}}+p_{q_{i}}\right)^{2}} \frac{\left\langle q_{i+1}, q_{i+2}\right\rangle^{2}}{\left\langle\bar{q}_{i+1}, q_{i+1}\right\rangle\left\langle\bar{q}_{i+2}, q_{i+2}\right\rangle} \\
& \times \frac{\left\langle q_{i+2}, \bar{q}_{i+1}\right\rangle}{\left\langle q_{i+2},\left(\bar{q}_{i} q_{i}\right)\right\rangle\left\langle\left(\bar{q}_{i} q_{i}\right), \bar{q}_{i+1}\right\rangle},
\end{aligned}
$$

and

$$
\begin{aligned}
\tilde{A}^{i}= & -\frac{\left\langle q_{i},\left(\bar{q}_{i} \bar{q}_{i+1}\right)\right\rangle^{2}}{\left\langle\bar{q}_{i}, q_{i}\right\rangle\left\langle\bar{q}_{i+1},\left(\bar{q}_{i} q_{i}\right)\right\rangle} \frac{1}{\left(p_{\bar{q}_{i}}+p_{q_{i}}+p_{\bar{q}_{i+1}}\right)^{2}} \\
& \times \frac{\left\langle q_{i+1}, q_{i+2}\right\rangle^{2}}{\left\langle\left(\bar{q}_{i+2} q_{i+2}\right), q_{i+1}\right\rangle\left\langle\bar{q}_{i+2}, q_{i+2}\right\rangle}
\end{aligned}
$$

Here the expression $\langle(i j), k\rangle$ is defined as $\left\langle\lambda_{p_{i}+p_{j}}, \lambda_{k}\right\rangle$, and the indices are understood mod 3.

From the momentum conservation we get $\lambda_{q_{i}} \phi_{q_{i}}+\lambda_{\bar{q}_{i}} \phi_{\bar{q}_{i}}+\lambda_{p_{q_{i}}+p_{\bar{q}_{i}}}=0$, so $\left\langle q_{i},\left(\bar{q}_{i} q_{i}\right)\right\rangle=\phi_{\bar{q}_{i}}\left\langle\bar{q}_{i}, q_{i}\right\rangle$ then

$$
\frac{\left\langle q_{i},\left(\bar{q}_{i} q_{i}\right)\right\rangle^{2}}{\left\langle\bar{q}_{i}, q_{i}\right\rangle} \frac{1}{\left(p_{\bar{q}_{i}}+p_{q_{i}}\right)^{2}}=\frac{\phi_{\bar{q}_{i}}^{2}\left\langle\bar{q}_{i}, q_{i}\right\rangle}{\left(p_{\bar{q}_{i}}+p_{q_{i}}\right)^{2}}=\frac{\phi_{\bar{q}_{i}}^{2}}{\left[\bar{q}_{i}, q_{i}\right]} .
$$

Using this result, we can write $A^{i}$ as

$$
A^{i}=\frac{\phi_{\bar{q}_{i}}^{2}}{\left[\bar{q}_{i}, q_{i}\right]} \frac{\left\langle q_{i+1}, q_{i+2}\right\rangle^{2}}{\left\langle\bar{q}_{i+1}, q_{i+1}\right\rangle\left\langle\bar{q}_{i+2}, q_{i+2}\right\rangle} \frac{\left\langle q_{i+2}, \bar{q}_{i+1}\right\rangle}{\left\langle q_{i+2},\left(\bar{q}_{i} q_{i}\right)\right\rangle\left\langle\left(\bar{q}_{i} q_{i}\right), \bar{q}_{i+1}\right\rangle} .
$$


Similarly, when $h_{1}=h_{2}=-1 / 2, h_{3}=1 / 2$, the result given by the CSW rules is

$$
A_{6}^{C S W}\left(\Lambda_{q_{1}}^{-}, \Lambda_{\bar{q}_{2}}^{+}, \Lambda_{q_{2}}^{-}, \Lambda_{\overline{q_{3}}}^{-}, \Lambda_{q_{3}}^{+}, \Lambda_{\bar{q}_{1}}^{+}\right)=\sum_{i=1}^{3} A^{i}+\sum_{i=1}^{3} \tilde{A}^{i}
$$

Now

$$
\begin{aligned}
& A^{1}=-\frac{\phi_{\bar{q}_{1}}^{2}}{\left[\bar{q}_{1}, q_{1}\right]} \frac{\left\langle q_{2}, \bar{q}_{3}\right\rangle^{2}}{\left\langle\bar{q}_{2}, q_{2}\right\rangle\left\langle\bar{q}_{3}, q_{3}\right\rangle} \frac{\left\langle q_{3}, \bar{q}_{2}\right\rangle}{\left\langle q_{3},\left(\bar{q}_{1} q_{1}\right)\right\rangle\left\langle\left(\bar{q}_{1} q_{1}\right), \bar{q}_{2}\right\rangle}, \\
& A^{2}=-\frac{\phi_{\bar{q}_{2}}^{2}}{\left[\bar{q}_{2}, q_{2}\right]} \frac{\left\langle q_{1}, \bar{q}_{3}\right\rangle^{2}}{\left\langle\bar{q}_{3}, q_{3}\right\rangle\left\langle\bar{q}_{1}, q_{1}\right\rangle} \frac{\left\langle q_{1}, \bar{q}_{3}\right\rangle}{\left\langle q_{1},\left(\bar{q}_{2} q_{2}\right)\right\rangle\left\langle\left(\bar{q}_{2} q_{2}\right), \bar{q}_{3}\right\rangle}, \\
& A^{3}=-\frac{\phi_{q_{3}}^{2}}{\left[\bar{q}_{3}, q_{3}\right]} \frac{\left\langle q_{1}, q_{2}\right\rangle^{2}}{\left\langle\bar{q}_{1}, q_{1}\right\rangle\left\langle\bar{q}_{2}, q_{2}\right\rangle} \frac{\left\langle q_{2}, \bar{q}_{1}\right\rangle}{\left\langle q_{2},\left(\bar{q}_{3} q_{3}\right)\right\rangle\left\langle\left(\bar{q}_{3} q_{3}\right), \bar{q}_{1}\right\rangle},
\end{aligned}
$$

and

$$
\begin{aligned}
\tilde{A}^{1}= & \frac{\left\langle\left(q_{3} \bar{q}_{1}\right), q_{1}\right\rangle^{2}}{\left\langle\bar{q}_{1}, q_{1}\right\rangle\left\langle\left(\bar{q}_{1} q_{1}\right), q_{3}\right\rangle} \frac{1}{\left(p_{\bar{q}_{2}}+p_{q_{2}}+p_{\bar{q}_{3}}\right)^{2}} \\
& \times \frac{\left\langle q_{2}, \bar{q}_{3}\right\rangle^{2}}{\left\langle\bar{q}_{2}, q_{2}\right\rangle\left\langle\bar{q}_{3},\left(\bar{q}_{2} q_{2}\right)\right\rangle}, \\
\tilde{A}^{2}= & \frac{\left\langle\left(q_{3} \bar{q}_{1}\right), \bar{q}_{3}\right\rangle^{2}}{\left.\left\langle\bar{q}_{3}, q_{3}\right\rangle\left\langle\bar{q}_{1}, \bar{q}_{3} q_{3}\right)\right\rangle} \frac{1}{\left(p_{\bar{q}_{3}}+p_{q_{3}}+p_{\bar{q}_{1}}\right)^{2}} \\
& \times \frac{\left\langle q_{1}, q_{2}\right\rangle^{2}}{\left\langle\bar{q}_{2}, q_{2}\right\rangle\left\langle\left(\bar{q}_{2} q_{2}\right), q_{1}\right\rangle}, \\
\tilde{A}^{3}= & \frac{\left\langle q_{1},\left(\bar{q}_{1} \bar{q}_{2}\right)\right\rangle^{2}}{\left\langle\bar{q}_{1}, q_{1}\right\rangle\left\langle\bar{q}_{2},\left(\bar{q}_{1} q_{1}\right)\right\rangle} \frac{1}{\left(p_{\bar{q}_{1}}+p_{q_{1}}+p_{\bar{q}_{2}}\right)^{2}} \\
& \times \frac{\left\langle q_{2}, \bar{q}_{3}\right\rangle^{2}}{\left\langle\bar{q}_{3}, q_{3}\right\rangle\left\langle\left(\bar{q}_{3} q_{3}\right), q_{2}\right\rangle} .
\end{aligned}
$$



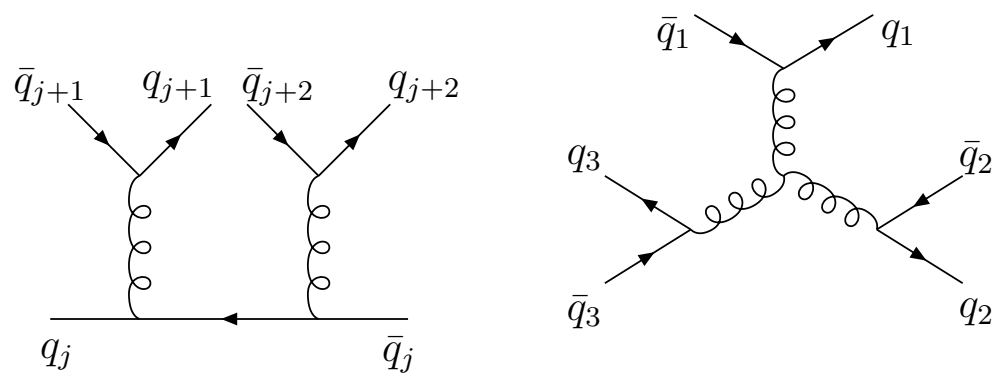

Figure 4: The 2 different kinds of Feynman diagrams contributing to the purely fermionic amplitude with 3 quark-antiquark pairs. In the left diagram $j$ can take 1,2,3 and the indices are understood as mod 3. So totally there are 4 diagrams.

Now we will compute this amplitudes by using the Feynman rules ${ }^{2}$. There are four Feynman diagrams as shown in Fig. 4. We will use the helicity trick (see, for example, [8] $)^{3}$.

The result for $h_{1}=h_{2}=h_{3}=-1 / 2$ is

$$
A_{6}^{\text {Feynman }}\left(\Lambda_{q_{1}}^{-}, \Lambda_{\bar{q}_{2}}^{+}, \Lambda_{q_{2}}^{-}, \Lambda_{\bar{q}_{3}}^{+}, \Lambda_{q_{3}}^{-}, \Lambda_{\bar{q}_{1}}^{+}\right)=\sum_{j=1}^{3} A^{j}+\tilde{A}
$$

where ${ }^{4}$

$$
\begin{aligned}
A^{j}= & -\left\langle p_{q_{j}}^{-}\left|\frac{i}{\sqrt{2}} \gamma^{\mu} i\left(p_{q_{j}}^{\rho}+p_{\bar{q}_{j+1}}^{\rho}+p_{q_{j+1}}^{\rho}\right) \gamma_{\rho} \frac{i}{\sqrt{2}} \gamma^{\nu}\right| p_{\bar{q}_{j}}^{-}\right\rangle \\
& \times\left\langle p_{q_{j+1}}^{-}\left|\frac{i}{\sqrt{2}} \gamma_{\mu}\right| p_{\bar{q}_{j+1}}^{-}\right\rangle\left\langle p_{q_{j+2}}^{-}\left|\frac{i}{\sqrt{2}} \gamma_{\nu}\right| p_{\bar{q}_{j+2}}^{-}\right\rangle \frac{-i}{\left(p_{\bar{q}_{j+1}}+p_{q_{j+1}}\right)^{2}} \frac{-i}{\left(p_{\bar{q}_{j+2}}+p_{q_{j+2}}\right)^{2}} \\
& \times \frac{1}{\left(p_{q_{j}}+p_{\bar{q}_{j+1}}+p_{q_{j+1}}\right)^{2}} \\
= & i \frac{\left\langle q_{j}, q_{j+1}\right\rangle\left[\bar{q}_{j}, \bar{q}_{j+2}\right]}{\left(p_{\bar{q}_{j+1}}+p_{q_{j+1}}\right)^{2}\left(p_{\bar{q}_{j+2}}+p_{q_{j+2}}\right)^{2}\left(p_{q_{j}}+p_{\bar{q}_{j+1}}+p_{q_{j+1}}\right)^{2}}
\end{aligned}
$$

\footnotetext{
${ }^{2}$ These calculations have been done in 37 . We thank Zvi Bern for reminding us of this.

${ }^{3}$ We note that the convention for $[i, j]$ in $[8$ is different from the one we used here by an extra -.

${ }^{4}$ The amplitudes we calculate are $A_{6}\left(\Lambda_{q_{1}}^{h_{1}}, \Lambda_{\bar{q}_{2}}^{-h_{2}}, \Lambda_{q_{2}}^{h_{2}}, \Lambda_{\bar{q}_{3}}^{-h_{3}}, \Lambda_{q_{3}}^{h_{3}}, \Lambda_{\bar{q}_{1}}^{-h_{1}}\right)$ instead of $A_{6}\left(\Lambda_{\bar{q}_{1}}^{-h_{1}}, \Lambda_{q_{1}}^{h_{1}}, \Lambda_{\bar{q}_{2}}^{-h_{2}}, \Lambda_{q_{2}}^{h_{2}}, \Lambda_{\bar{q}_{3}}^{-h_{3}}, \Lambda_{q_{3}}^{h_{3}}\right)$, this fact gives an extra -.
} 


$$
\times\left(\left\langle q_{j}, q_{j+2}\right\rangle\left[q_{j}, \bar{q}_{j+1}\right]+\left\langle q_{j+1}, q_{j+2}\right\rangle\left[q_{j+1}, \bar{q}_{j+1}\right]\right),
$$

and

$$
\begin{aligned}
\tilde{A}= & -\left\langle p_{q_{1}}^{-}\left|\frac{i}{\sqrt{2}} \gamma_{\mu}\right| p_{\bar{q}_{1}}^{-}\right\rangle\left\langle p_{q_{2}}^{-}\left|\frac{i}{\sqrt{2}} \gamma_{\nu}\right| p_{\bar{q}_{2}}^{-}\right\rangle\left\langle p_{q_{3}}^{-}\left|\frac{i}{\sqrt{2}} \gamma_{\rho}\right| p_{\bar{q}_{3}}^{-}\right\rangle \\
& \times \frac{i}{\sqrt{2}}\left(2\left(k_{\bar{q}_{1}}+k_{q_{1}}\right)_{\rho} \eta_{\mu \nu}+2\left(k_{\bar{q}_{2}}+k_{q_{2}}\right)_{\mu} \eta_{\nu \rho}+2\left(k_{\bar{q}_{3}}+k_{q_{3}}\right)_{\nu} \eta_{\rho \mu}\right) \\
& \times \prod_{j=1}^{3} \frac{-i}{\left(p_{\bar{q}_{j}}+p_{q_{j}}\right)^{2}} \\
= & \frac{-i}{\prod_{j=1}^{3}\left(p_{\bar{q}_{j}}+p_{q_{j}}\right)^{2}} \sum_{k=1}^{3}\left\langle q_{k}, q_{k+1}\right\rangle\left[\bar{q}_{k}, \bar{q}_{k+1}\right] \\
& \times\left(\left\langle\bar{q}_{k}, q_{k+2}\right\rangle\left[\bar{q}_{k}, \bar{q}_{k+2}\right]+\left\langle q_{k}, q_{k+2}\right\rangle\left[q_{k}, \bar{q}_{k+2}\right]\right) .
\end{aligned}
$$

The bras and kets in eqs. (28) and (29) are denoted by the momenta of corresponding particles.

When $h_{1}=h_{2}=-1 / 2, h_{3}=1 / 2$, we can similarly obtain the following result by using the Feynman rules,

$$
A_{6}^{\text {Feynman }}\left(\Lambda_{q_{1}}^{-}, \Lambda_{\bar{q}_{2}}^{+}, \Lambda_{q_{2}}^{-}, \Lambda_{\bar{q}_{3}}^{-}, \Lambda_{q_{3}}^{+}, \Lambda_{\bar{q}_{1}}^{+}\right)=\sum_{j=1}^{3} A^{j}+\tilde{A} .
$$

Now

$$
\begin{aligned}
A^{1}= & i \frac{\left\langle q_{1}, q_{2}\right\rangle\left[\bar{q}_{1}, q_{3}\right]}{\left(p_{\bar{q}_{2}}+p_{q_{2}}\right)^{2}\left(p_{\bar{q}_{3}}+p_{q_{3}}^{2}\right)\left(p_{q_{1}}+p_{\bar{q}_{2}}+p_{q_{2}}\right)^{2}} \\
& \times\left(\left\langle q_{1}, \bar{q}_{3}\right\rangle\left[q_{1}, \bar{q}_{2}\right]+\left\langle q_{2}, \bar{q}_{3}\right\rangle\left[q_{2}, \bar{q}_{2}\right]\right), \\
A^{2}= & i \frac{\left\langle q_{2}, \bar{q}_{3}\right\rangle\left[\bar{q}_{2}, \bar{q}_{1}\right]}{\left(p_{\bar{q}_{3}}+p_{q_{3}}\right)^{2}\left(p_{\bar{q}_{1}}+p_{q_{1}}\right)^{2}\left(p_{q_{2}}+p_{\bar{q}_{3}}+p_{q_{3}}\right)^{2}} \\
& \times\left(\left\langle q_{2}, q_{1}\right\rangle\left[q_{2}, q_{3}\right]+\left\langle\bar{q}_{3}, q_{1}\right\rangle\left[\bar{q}_{3}, q_{3}\right]\right), \\
A^{3}= & i \frac{\left\langle\bar{q}_{3}, q_{2}\right\rangle\left[q_{3}, \bar{q}_{1}\right]}{\left(p_{\bar{q}_{1}}+p_{q_{1}}\right)^{2}\left(p_{\bar{q}_{2}}+p_{q_{2}}\right)^{2}\left(p_{q_{3}}+p_{\bar{q}_{1}}+p_{q_{1}}\right)^{2}} \\
& \times\left(\left\langle q_{3}, q_{1}\right\rangle\left[q_{3}, \bar{q}_{2}\right]+\left\langle\bar{q}_{1}, q_{1}\right\rangle\left[\bar{q}_{1}, \bar{q}_{2}\right]\right),
\end{aligned}
$$


and

$$
\begin{aligned}
\tilde{A}= & -i\left(\left\langle q_{1}, q_{2}\right\rangle\left[\bar{q}_{1}, \bar{q}_{2}\right]\left(\left\langle\bar{q}_{3}, \bar{q}_{1}\right\rangle\left[q_{3}, \bar{q}_{1}\right]+\left\langle\bar{q}_{3}, q_{1}\right\rangle\left[q_{3}, q_{1}\right]\right)\right. \\
& +\left\langle q_{2}, \bar{q}_{3}\right\rangle\left[\bar{q}_{2}, q_{3}\right]\left(\left\langle q_{1}, \bar{q}_{2}\right\rangle\left[\bar{q}_{1}, \bar{q}_{2}\right]+\left\langle q_{1}, q_{2}\right\rangle\left[\bar{q}_{1}, q_{2}\right]\right) \\
& \left.+\left\langle\bar{q}_{3}, q_{1}\right\rangle\left[q_{3}, \bar{q}_{1}\right]\left(\left\langle q_{2}, q_{3}\right\rangle\left[\bar{q}_{2}, q_{3}\right]+\left\langle q_{2}, \bar{q}_{3}\right\rangle\left[\bar{q}_{2}, \bar{q}_{3}\right]\right)\right) \\
& \times \frac{1}{\sum_{j=1}^{3}\left(p_{\bar{q}_{j}}+p_{q_{j}}\right)^{2}} .
\end{aligned}
$$

There are the following constrains from the momentum conservation:

$$
\sum_{i=1}^{3} \lambda_{q_{i}}^{\alpha} \tilde{\lambda}_{q_{i}}^{\dot{\alpha}}+\sum_{i=1}^{3} \lambda_{\bar{q}_{i}}^{\alpha} \tilde{\lambda}_{\bar{q}_{i}}^{\dot{\alpha}}=0, \alpha=1,2, \dot{\alpha}=1,2 .
$$

From these constrains, we can solve $\tilde{\lambda}_{\bar{q}_{1}}^{\dot{\alpha}}$ and $\tilde{\lambda}_{q_{1}}^{\dot{\alpha}}$ in terms of other $\lambda^{\prime}$ 's and $\tilde{\lambda}^{\prime}$ 's. The result is

$$
\begin{aligned}
& \tilde{\lambda}_{\bar{q}_{1}}^{\dot{\alpha}}=-\sum_{i=2}^{3} \frac{\tilde{\lambda}_{q_{i}}^{\dot{\alpha}}\left\langle\lambda_{q_{i}}, \lambda_{q_{1}}\right\rangle+\tilde{\lambda}_{\bar{q}_{i}}^{\dot{\alpha}}\left\langle\lambda_{\bar{q}_{i}}, \lambda_{q_{1}}\right\rangle}{\left\langle\lambda_{\bar{q}_{1}}, \lambda_{q_{1}}\right\rangle}, \\
& \tilde{\lambda}_{q_{1}}^{\dot{\alpha}}=-\sum_{i=2}^{3} \frac{\tilde{\lambda}_{q_{i}}^{\dot{\alpha}}\left\langle\lambda_{q_{i}}, \lambda_{\bar{q}_{1}}\right\rangle+\tilde{\lambda}_{\bar{q}_{i}}^{\dot{\alpha}}\left\langle\lambda_{\bar{q}_{i}}, \lambda_{\bar{q}_{1}}\right\rangle}{\left\langle\lambda_{q_{1}}, \lambda_{\bar{q}_{1}}\right\rangle} .
\end{aligned}
$$

We noted that we don't treat $\tilde{\lambda}$ as the complex conjugation of $\lambda$. So in fact, we have use analytic continuation to the spacetime with signature $(2,2)$, after we obtain our result we can go back the Minkowski space. By using these results and with the help of symbolic manipulation, we can find that

$$
A_{6}^{\text {Feynman }}=-i A_{6}^{C S W}
$$

either in the case when $h_{1}=h_{2}=h_{3}=-1 / 2$ or in the case when $h_{1}=h_{2}=$ $-1 / 2, h_{3}=1 / 2$. From the argument above we know that we can obtain the same result for all of the helicity configurations as promised.

\section{Acknowledgment}

We would like to thank Zvi Bern for useful discussion during ichep'04 at Beijing and Chuan-Jie Zhu for suggesting this topic and discussion. 


\section{References}

[1] E. Witten, "Perturbative Gauge Theory As A String Theory In Twistor Space," hep-th/0312171.

[2] R. Penrose, "Twistor Algebra," J. Math. Phys. 8 (1967) 345; "The Central Programme of Twistor Theory," Chaos, Solitons, and Fractal 10 (1999) 581.

[3] F. Cachazo, P. Svrcek and E. Witten, "MHV Vertices and Tree Amplitudes In Gauge Theory," JHEP 0409:006 (2004), hep-th/0403047.

[4] S. Parke and T. Taylor, "An Amplitude For N Gluon Scattering," Phys. Rev. Lett. 56 (1986) 2459.

[5] F. A. Behrends and W. T. Giele, "Recursive Calculations For Processes With $N$ Gluons," Nucl. Phys. B306 (1988) 759.

[6] M. L. Mangano and S. Parke, "Multiparton Amplitudes in Guage Theories," Phys. Rept. 200 (1991) 30.

[7] Z. Bern, "String-Based Perturbative Methods for Gauge Theories," TASI Lectures, 1992, hep-ph/9304249

[8] L. Dixon, "Calculating Scattering Amplitudes Efficiently," TASI Lectures, 1995, hep-ph/9601359

[9] Z. Bern, L. Dixon, and D. Kosower, "Progress In One-Loop QCD Calculations," Ann. Rev. Nucl. Part. Sci. 36 (1996) 109, hep-ph/9602280.

[10] C. Anastasiou, Z. Bern, L. Dixon, and D. Kosower, "Planar Amplitudes In Maximally Supersymmetric Yang-Mills Theory," Phys.Rev.Lett. 91 (2003) 251602, hep-th/0309040; Z. Bern, A. De Freitas, and L. Dixon, "Two Loop Helicity Amplitudes For Quark Gluon Scattering In QCD and Gluino Gluon Scattering In Supersymmetric Yang-Mills Theory," JHEP 0306:028 (2003), hep-ph/0304168.

[11] V. P. Nair, "A Current Algebra for Some Gauge Theory Amplitudes," Phsy. Lett. B214 (1988) 215-218.

[12] J.-B. Wu and C.-J. Zhu, " MHV Vertices and Fermionic Scattering Amplitudes in Gauge Theory with Quarks and Gluinos," hep-th/0406146. 
[13] C.-J. Zhu, "The Googly Amplitudes in Gauge theory," JHEP 0404:032 (2004), hep-th/0403115

[14] J.-B. Wu and C.-J. Zhu, "MHV Vertices and Scattering Amplitudes in Gauge Theory," JHEP 0407:032 (2004), hep-th/0406085.

[15] R. Roiban, M. Spradlin, and A. Volovich, "A Googly Amplitude From The B Model In Twistor Space," JHEP 0404:012 (2004), hep-th/0402016, R. Roiban and A. Volovich, "All Googly Amplitudes From The B Model In Twistor Space," hep-th/0402121.

[16] R. Roiban, M. Spradlin, and A. Volovich, "On the Tree-Level S-Matrix of Yang-Mills Theory ," Phys.Rev. D70 (2004) 026009, hep-th/0403190.

[17] N. Berkovits, "An Alternative String Theory in Twistor Space for $N=4$ Super-Yang-Mills," Phys.Rev.Lett. 93 (2004) 011601, hep-th/0402045.

[18] N. Berkovits and L. Motl, "Cubic Twistorial String Field Theory," J. High Energy Phys. 0404 (2004) 056, hep-th/0403187.

[19] M. Aganagic and C. Vafa, "Mirror Symmetry and Supermanifolds," hep-th/0403192.

[20] E. Witten, "Parity Invariance For Strings In Twistor Space," hep-th/0403199.

[21] A. Neitzke and C. Vafa, " $N=2$ Strings and the Twistorial CalabiYau," hep-th/0402128

[22] N. Nekrasov, H. Ooguri and C. Vafa, "S-duality and Topological Strings," hep-th/0403167.

[23] G. Georgiou and V. V. Khoze, "Tree Amplitudes in Gauge Theory as Scalar MHV Diagrams," JHEP 0405 (2004) 070, hep-th/0404072

[24] S. Gukov, L. Motl and A. Neitzke, "Equivalence of twistor prescriptions for super Yang-Mills," hep-th/0404085.

[25] W. Siegel, "Untwisting the twistor superstring," hep-th/0404255. 
[26] S. Giombi, R. Ricci, D. Robles-Llana and D. Trancanelli, "A Note on Twistor Gravity Amplitudes," JHEP 0407 (2004) 059, hep-th/0405086.

[27] A. D. Popov and C. Saemann, "On Supertwistors, the Penrose-Ward Transform and N=4 super Yang-Mills Theory," hep-th/0405123.

[28] N. Berkovits and E. Witten, "Conformal Supergravity in TwistorString Theory," JHEP 0408:009 (2004), hep-th/0406051.

[29] I. Bena, Z. Bern and D. A. Kosower, "Twistor-Space Recursive Formulation of Gauge-Theory Amplitudes," hep-th/0406133.

[30] D. A. Kosower, "Next-to-Maximal Helicity Violating Amplitudes in Gauge Theory," hep-th/0406175.

[31] F. Cachazo, P. Svrcek and E. Witten, "Twistor Space Structure of One-Loop Amplitudes in Gauge Theory," hep-th/0406177.

[32] A. D. Popov and M. Wolf, "Topological B-model on weighted projective spaces and self-dual models in four dimensions," JHEP 0409:007 (2004), hep-th/0406224.

[33] G. Georgiou, E. W. N. Glover and V. V. Khoze, "Non-MHV Tree Amplitudes in Gauge Theory," JHEP 0407:048 (2004), hep-th/0407027.

[34] A. Brandhuber, B. Spence and G. Travaglini, "One-loop gauge theory amplitudes in $\mathrm{N}=4$ super Yang-Mills from MHV vertices," hep-th/0407214.

[35] I. Bars, "Twistor superstring in 2T-physics," hep-th/0407239.

[36] Y. Abe, V. P. Nair and M. I. Park, "Multigluon amplitudes, N = 4 constraints and the WZW model," hep-th/0408191.

[37] J. F. Gunion and Z. Kunszt, "Six-Quark Subprocesses in QCD," Phys. Lett. B176 (1986) 163.

[38] M. T. Grisaru, H. N. Pendleton and P. van Nieuwenhuizen, "Supergravity and the S Matrix," Phys. Rev. D15(1977) 996.

[39] M. T. Grisaru and H. N. Pendleton, "Some Properties of Scattering Amplitudes in Supersymmetric Theories," Nucl. Phys. B214(1977) 81. 
[40] M. Mangano and S. J. Parke, "Quark-Gluon Amplitudes in the Dual Expansion", Nucl. Phys. B299 (1988) 673.

[41] M. Mangano, "The Color Structure of Gluon Emission," Nucl. Phys. B309 (1988) 461. 